\title{
BMJ Open Online versus face-to-face pulmonary rehabilitation for patients with chronic obstructive pulmonary disease: randomised controlled trial
}

\author{
Simon Bourne, ${ }^{1,2}$ Ruth DeVos, ${ }^{1,2}$ Malcolm North, ${ }^{2}$ Anoop Chauhan, ${ }^{1}$ Ben Green, ${ }^{1}$ \\ Thomas Brown, ${ }^{1}$ Victoria Cornelius, ${ }^{3}$ Tom Wilkinson ${ }^{2,4}$
}

To cite: Bourne S, DeVos R, North M, et al. Online versus face-to-face pulmonary rehabilitation for patients with chronic obstructive pulmonary disease: randomised controlled trial. BMJ Open 2017;7:e014580. doi:10.1136/ bmjopen-2016-014580

- Prepublication history and additional material are available. To view these files, please visit the journal online (http://dx.doi. org/10.1136/bmjopen-2016014580).

Received 4 October 2016 Revised 10 May 2017 Accepted 18 May 2017

CrossMark

${ }^{1}$ Portsmouth Hospitals NHS Trust, Portsmouth, UK ${ }^{2}$ myMHealth Ltd Bournemouth, UK

${ }^{3}$ Imperial College, London, UK ${ }^{4}$ Clinical and Experimental Sciences, Faculty of Medicine, University of Southampton, Southampton, UK

Correspondence to

Dr Tom Wilkinson; t.wilkinson@ soton.ac.uk

\section{ABSTRACT}

Objective To obtain evidence whether the online pulmonary rehabilitation(PR) programme 'my-PR' is non-inferior to a conventional face-to-face PR in improving physical performance and symptom scores in patients with COPD. Design A two-arm parallel single-blind, randomised controlled trial.

Setting The online arm carried out pulmonary rehabilitation in their own homes and the face to face arm in a local rehabilitation facility.

Participants 90 patients with a diagnosis of chronic obstructive pulmonary disease (COPD), modified Medical Research Council score of 2 or greater referred for pulmonary rehabilitation $(P R)$, randomised in a 2:1 ratio to online $(n=64)$ or face-to-face PR $(n=26)$. Participants unable to use an internet-enabled device at home were excluded.

Main outcome measures Coprimary outcomes were 6 min walk distance test and the COPD assessment test (CAT) score at completion of the programme.

Interventions A 6-week PR programme organised either as group sessions in a local rehabilitation facility, or online PR via log in and access to 'myPR'.

Results The adjusted mean difference for the 6 min walk test (6MWT) between groups for the intention-to-treat (ITT) population was $23.8 \mathrm{~m}$ with the lower $95 \% \mathrm{Cl}$ well above the non-inferiority threshold of $-40.5 \mathrm{~m}$ at $-4.5 \mathrm{~m}$ with an upper $95 \% \mathrm{Cl}$ of $+52.2 \mathrm{~m}$. This result was consistent in the per-protocol (PP) population with a mean adjusted difference of $15 \mathrm{~m}(-13.7$ to 43.8$)$. The CAT score difference in the ITT was -1.0 in favour of the online intervention with the upper $95 \% \mathrm{Cl}$ well below the non-inferiority threshold of 1.8 at 0.86 and the lower $95 \% \mathrm{Cl}$ of -2.9 . The PP analysis was consistent with the ITT.

Conclusion PR is an evidenced-based and guidelinemandated intervention for patients with COPD with functional limitation. A 6-week programme of onlinesupported PR was non-inferior to a conventional model delivered in face-to-face sessions in terms of effects on 6MWT distance, and symptom scores and was safe and well tolerated.

\section{INTRODUCTION}

Chronic obstructive pulmonary disease (COPD) is a highly prevalent condition, which results in gradual loss of lung

\section{Strengths and limitations of this study}

- This study explored the efficacy and safety 'myPR', a novel method for delivering pulmonary rehabilitation by online support compared with conventional faceto-face delivery in classes using a randomised controlled trial to explore whether the online programme was non-inferior to the standard model.

Due to the nature of the intervention, only patients with access to the internet at home could be included in the study.

- Further limitations of this study include the limited sample size, and the absence of long-term followup. Larger studies are required to explore the healtheconomic benefits of this model and applicability in different healthcare settings.

function, increasing symptoms and functional limitation over time. ${ }^{1}$ Pulmonary rehabilitation (PR) is a non-pharmacological intervention at the core of management of COPD, aimed at reducing the burden of symptoms by increasing exercise tolerance and improving self-management. With an established evidence-base, PR has been placed at the centre of interventions for COPD and its provision is mandated by the National Institute for Health and Care Excellence (NICE) as a key pillar of integrated care. ${ }^{1}$ The model of care for providing PR is traditionally a face-to-face, structured programme of exercise training and education completed in a supervised, centre-based setting over an established protocol of a minimum of 6 weeks. ${ }^{2}$

While PR has been shown to improve functional performance and physical activity ${ }^{3}$ and greater activity levels have themselves been associated with reduced risk of hospitalisation, ${ }^{4}$ access to programmes can be problematic for some patients and the impact of conventional PR is limited by 
suboptimal attendance and high dropout rates. ${ }^{56}$ With an ever-increasing burden on services, conventional models of care are constantly being challenged and alternative, cost-effective ways of delivering healthcare to a larger cohort of patients are being sought. Indeed, the key message and goal in the recent American Thoracic Society/European. Respiratory Society policy statement of the implementation and delivery of PR is 'to expand provision of PR to suitable patients worldwide'.

Patients with COPD are encouraged to carry out PR exercises in their home environment, and even unsupervised exercise has been shown to be an effective way of increasing exercise tolerance. ${ }^{8}$ More recently, the use of Telehealth has been trialled as an alternative, and innovative way of delivering PR to individuals in their home, with aims to increase its uptake and, in particular access for those in isolated areas or who have transport issues. This home-based intervention using tele-monitoring equipment has shown some promise in maintaining and further improving physical capacity but hardware-related costs are high. ${ }^{9}{ }^{10}$ In 2015, 86\% of patients with chronic cardiopulmonary disease had internet access, ${ }^{11}$ and with this ever-increasing presence of technology in homes, online PR is beginning to emerge as an alternative way of delivering PR. ${ }^{12}$ Indeed, the current British Thoracic Society (BTS) PR guidelines ${ }^{2}$ recognise that 'technology has the potential to be used as an adjunct to rehabilitation or even provide a "rehabilitative" service'.

Although aspects of PR have been delivered in the home setting, the documented attempts have, to date, lacked the capability of administering a comprehensive programme provided by conventional face-to-face PR. The conventional model combines delivery of educational component including information on the condition, prescribed treatments and advice on exercise and self-directed care, delivered alongside an incremental exercise programme. The educational component of PR is recognised by the BTS as 'fundamentally integral to the format and success of the programme' and 'the intention of the educational element is to support the lifestyle and behavioural change and assist self-management to promote self-efficacy'. ${ }^{2}$

In response to the recognised demand for alternative ways of delivering PR, a comprehensive, online internet-based PR programme consisting of a 6-week incremental exercise programme alongside education sessions was developed for patients with COPD. This online PR programme, known as myPR, was developed by a multidisciplinary team of respiratory specialists and is designed to mirror all of the components of a conventional COPD PR programme.

We conducted a non-inferiority randomised controlled clinical trial to compare efficacy and safety of PR supported by the online application compared with a face-to-face class-based PR programme.

\section{METHODS}

\section{Study design}

This was a prospective, parallel group, single-blind randomised controlled trial conducted in a single centre in the UK. Patients were recruited from a range of primary and secondary care clinical settings consistent with the route of referral for PR. The non-inferiority trial design was to compare the clinical delivery of a 6 -week online $\mathrm{PR}$ programme (myPR) to the current clinical standard of face-to-face PR programme delivered in a conventional community setting, to patients with COPD. This study was approved by the research ethics committee for Berkshire B of the UK Health Research Authority (15/SC/0345). The study was registered online as NCT02706613.

Patients were consulted and played an active role in the development of mycopd-the online intervention platform. Patients were involved in reviewing the design of the study including the potential study burden on people with COPD, the choice and format of patient-reported outcome measures, the writing of the patient information sheets and the consent form.

We randomised eligible patients with COPD using a computerised block permutation randomise sequencer in a ratio of 2:1 to either the online arm (myPR) or to receive standard face-to-face PR. A 2:1 ratio was used to reduce the number of subjects in the more costly faceto-face arm while maintaining power. Randomisation was stratified by severity of COPD (forced expiratory volume in $1 \mathrm{~s}\left(\mathrm{FEV}_{1}\right) \%$ predicted) to ensure equal distribution in both arms and used an online system for concealed allocation.

Outcomes were measured at baseline and within 1 week of completion of either arm of the study. Due to the nature of the intervention, blinding of participants was not possible. Study staff carrying out the postintervention assessments (outcome assessors) were blind to which arm the patient had been randomised to.

\section{Study population}

Participants suitable for PR were recruited to the study from Portsmouth Hospitals NHS Trust outpatient respiratory clinics. All those participants wishing to participate were issued with a Patient Invitation Letter and a Patient Information Sheet. Inclusion criteria were a diagnosis of COPD as defined by the NICE COPD guidelines with a modified Medical Research Council (mMRC) dyspnoea of grade 2 or greater, with access to the internet and the ability to operate a web platform and aged 40 years or greater. Exclusion criteria were an exacerbation requiring additional antibiotics and/or steroids within 2 weeks prior to screening; patients who had already undertaken a PR programme within the last 6 months; patients who have another respiratory disease as their main complaint other than COPD; uncontrolled hypertension; unstable cardiovascular disease or significant desaturation that would make PR exercise unsafe or prevent programme participation; patients who were unable to walk or whose ability to walk safely and independently is significantly impaired 
due to non-respiratory-related conditions and/or cognitive impairment; patients who are unable to read or use an internet-enabled device or do not have access to the internet at home and a 'Timed Up and Go' (TUG) test $>4 \mathrm{~s}$. The TUG test was introduced as a way of assessing safety of unsupervised exercise of patients completing the $\mathrm{PR}$ programme in their homes, as it is a reliable and valid test for quantifying functional mobility ${ }^{12}$ and has been validated for use with COPD. ${ }^{13}$

\section{Online PR (myPR)}

After assessment, participants randomised to the online arm were issued with unique login details to access myPR. They were given basic instructions on the use of the programme, in a brief 5-10 min introductory session face to face with a member of the clinical research team. They were instructed to access myPR at least twice and up to five times a week. The initial start-up instructions on the programme were designed to explain each step of myPR to the patient, and further instructions were given as they progressed. The physiotherapist leading the online programme also delivered the face-to-face programmes to ensure standardisation between the programmes. Patients were advised to carry out exercises at a time that was convenient to them and when they felt their energy levels were at their best. No specific advice was given regarding exercise modification as this is built into the online programme itself.

The online programme is incremental in nature and ran over 6 weeks and each week the length of each of the 10 exercises increased by $30 \mathrm{~s}$, starting from $60 \mathrm{~s}$ in week 1 , to $3 \frac{1}{2}$ min in week 6 . The on-screen exercises were designed to be carried out with the patient in real time, with the patient following and keeping up with the video-facilitated exercises. One minute of rest time was given between each of the 10 exercises, with advice given on Borg score measurement along with other tips on managing breathlessness. During each of the 6 weeks of exercise, patients were directed to watch three different educational videos per week as education is a recognised and important component of PR and helps promote self-management. These educational sessions included anatomy of the lungs, an explanation of COPD, management of anxiety and depression, claiming benefits, self-management, managing breathlessness, medications and treatments, managing exacerbations of COPD, sputum clearance using the Active Cycle of Breathing Technique, nutrition, pacing, smoking cessation and advice on travel with COPD. All of these educational sessions are suggested in the current BTS PR guidelines, and patients could access these videos as often as they wished each week.

Contact details of the research team were provided so that patients had a point of reference for any queries they had regarding the technology or any health concerns. Details of the online programme can be accessed via www. mymhealth.com/mycopd.

\section{Face-to-face PR}

Patients randomised to the conventional face-to-face PR were given the dates and times of the next available PR being run in a rehabilitation facility by a physiotherapist and nurse on the research team. Patients attended two supervised sessions for 6 weeks and were asked to carry out exercises at home an additional three times per week. The programme consisted of 10 exercise stations, which were identical to the exercises carried out by the patients using myPR. The 10 exercises included biceps curls, squats, push ups against a wall, leg extensions in a sitting position, upright row with weights, sit-to-stand, arm swings with a stick, leg kicks to the side, arm punches with weights and step-ups. Both the online and face-toface programmes also included warm up and cool down sessions.

The same educational sessions as on myPR were then delivered, but were presented and discussed orally rather than in video format as in myPR, which offered patients the opportunity to address questions.

\section{Primary and secondary outcomes}

The primary outcome measures were to compare best performance $6 \mathrm{~min}$ walk distance $(6 \mathrm{MWD})$ test over a $30 \mathrm{~m}$ course on completion of the online and conventional PR programmes using the 6 min walk test (6MWT) performed according to national standards, ${ }^{2}$ and impact on health status using the COPD assessment test (CAT) score. Secondary outcome measures included the St Georges Respiratory Questionnaire (SGRQ) to assess respiratory quality of life, and the Hospital Anxiety and Depression Scale (HADS) to assess anxiety and depression. Safety was assessed by the incidence of adverse events (AEs) in each arm at study completion.

\section{Adverse events}

AEs were captured in the face-to-face group at the start of each session (twice a week) during the 6-week intervention and at final assessment. In the online arm, AEs were captured during a weekly phone call to the participant from the study clinical team and at final assessment. Causality and severity was assessed by the clinical study team.

\section{STATISTICAL ANALYSES}

\section{Sample size calculation}

The size of the study was chosen with consideration to provision of preliminary evidence for the non-inferiority of online PR as compared with gold standard face-to-face PR. As a result, the primary focus was to obtain an estimate for the lower bound of the 95\% CI for the 6MWD test, and upper bound of the $90 \%$ CI for the CAT. In a fully powered study, it is common that the non-inferiority margin is set to be half the clinically important minimum difference. As this is the first examination of the intervention the non-inferiority margin was chosen to be less than the minimum important clinical difference but not as high as the commonly used criteria of half the difference. 
From the published literature and practice guidelines on the 6MWD test, $54 \mathrm{~m}$ was a widely accepted minimum value of a meaningful increase in patient's perception of exercise performance. ${ }^{1415}$ This level was originally used to establish power and calculate the appropriate sample size. Assuming no difference between intervention arms and a SD of $100,{ }^{17}$ we required 75 participants (2:1 ratio) to estimate the lower $95 \% \mathrm{CI}$ bound for the mean difference to be no more than $40.5 \mathrm{~m}$. Subsequent to the study design, an update minimally clinically important difference of 30 $\mathrm{m}$ has been proposed and adopted. ${ }^{16}{ }^{17}$ Consideration of both cut-offs was undertaken in the analysis.

An accepted clinically important minimum difference of the CAT score is estimated to be 1.8 with a SD of $6.4 .^{20}$ Assuming no difference between intervention arms and a SD of 6.4, we required 94 participants (2:1 ratio) to estimate the upper $90 \%$ CI bound for the mean difference to be no more than 1.8. We took the larger of these two values ( $\mathrm{n}=94$ : 63:32 per arm).

\section{Randomisation}

Participants were randomised using permuted blocks via an online randomisation system hosted by myMHealth in a ratio of 2:1 with more participants being randomised to the online myPR arm. A concealed allocation was performed. Randomisation was stratified by disease severity defined by the global initiative for obstructive lung disease (GOLD) classification of COPD severity. ${ }^{18}$

\section{Blinding}

To ensure the study team remained blind as to which arm of the study each participant was on, they were divided into two teams. One team was responsible for the assessment and randomisation of participants onto the study and the other team provided the after-intervention assessment. A separate team member, who was not involved in the prestudy or poststudy assessments, was not blinded, to ensure availability to answer any questions participants had throughout the study, and deal with any potential adverse events. All subjects were asked in advance not to discuss their PR programme during assessments.

\section{Statistical methods}

Statistical analysis was performed for both the intention-to-treat (ITT) population and per-protocol (PP) population. ITT analysis included all participants in the arms they were randomised to regardless of adherence to either intervention. The frequency, patterns and predictors of missing data were explored. Data at follow-up was imputed regardless of the reason for missing. Multiple

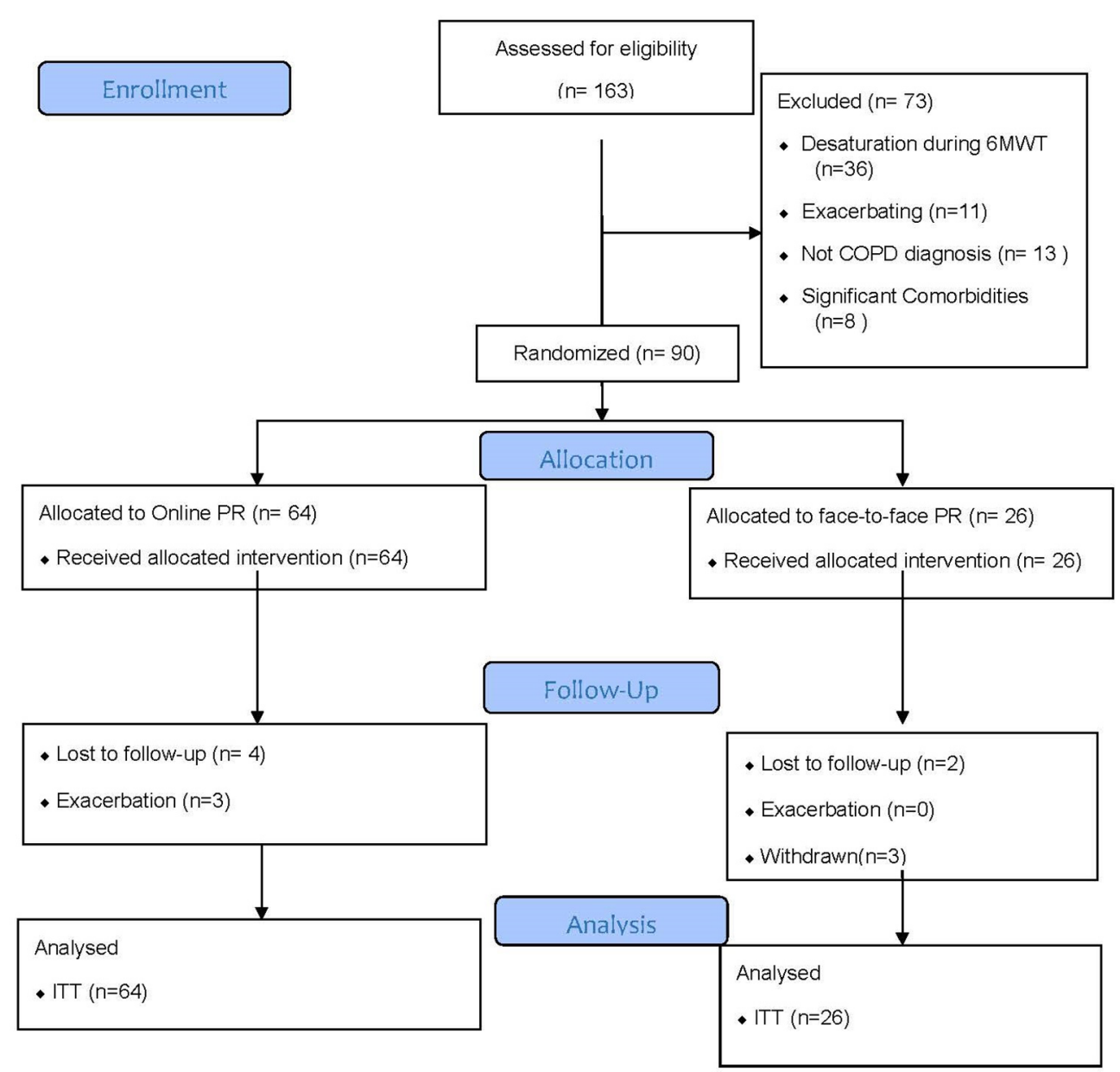

Figure 1 CONSORT diagram patient flow in study. COPD, chronic obstructive pulmonary disease; ITT, intention to treat; PR, pulmonary rehabilitation. 


\begin{tabular}{|c|c|c|}
\hline Baseline variables & $\begin{array}{l}\text { Face-to-face } \\
\text { PR }(n=26)\end{array}$ & $\begin{array}{l}\text { Online PR } \\
(n=64)\end{array}$ \\
\hline Age (years), mean (SD) & $71.4(8.6)$ & $69.1(7.9)$ \\
\hline Gender (male), n (\%) & $18(69)$ & $41(62)$ \\
\hline \multicolumn{3}{|l|}{ Smoking, n (\%) } \\
\hline Current smoker & $6(23)$ & $9(14)$ \\
\hline Ex-smoker & $20(76.9)$ & 55 (85.9) \\
\hline \multicolumn{3}{|l|}{ COPD severity, n (\%) } \\
\hline Mild & $5(19)$ & $15(23)$ \\
\hline Moderate & $13(50)$ & $26(41)$ \\
\hline Severe & $7(27)$ & $17(27)$ \\
\hline Very severe & $1(4)$ & $6(9)$ \\
\hline $\mathrm{FEV}_{1}$, mean (SD) & $1.66(0.67)$ & $1.63(0.71)$ \\
\hline $\mathrm{FEV}_{1} \%$ predicted, mean (SD) & $60.5(20.1)$ & $58.0(23.6)$ \\
\hline FVC, mean (SD) & $2.97(1.03)$ & $3.03(0.99)$ \\
\hline FVC predicted, mean (SD) & $83.2(21.2)$ & $88.4(22.0)$ \\
\hline
\end{tabular}

COPD, chronic obstructive pulmonary disease; FEV1, forced expiratory volume in $1 \mathrm{~s}$; FVC, forced vital capacity; PR, pulmonary rehabilitation.

imputation was implemented based on chained equation model and using age, gender, baseline scores and COPD severity assuming unobserved measurements were missing at random (100 datasets). ${ }^{19}$ Analyses were repeated for participants with complete data only and compared with analyses where missing data were imputed.

In the gold standard care arm, participants were invited to two face-to-face sessions per week. In the intervention arm, participants could access rehabilitation programme as much as they wanted to per week, although they were requested to access the programme

\begin{tabular}{lll}
\hline Table 2 Comorbidities by intervention arm \\
\hline Comorbidities & \multicolumn{2}{l}{ Face-to-face } \\
$\mathbf{n}(\%)$ & Online n (\%) \\
\hline Hypertension & $7(26.9 \%)$ & $23(35.9 \%)$ \\
\hline Cardiovascular disease & $13(50 \%)$ & $22(34.3 \%)$ \\
\hline Cerebrovascular disease & $1(3.8 \%)$ & $5(7.8 \%)$ \\
\hline Dermatological & 0 & $7(10.9 \%)$ \\
\hline Diabetes and endocrine & $6(23.1 \%)$ & $14(21.9 \%)$ \\
\hline Gastroenterological & $5(19.2 \%)$ & $21(32.8 \%)$ \\
\hline Haematological & $1(3.8 \%)$ & $1(1.6 \%)$ \\
Neurological and psychiatric & $3(11.5 \%)$ & $11(17.2 \%)$ \\
\hline History of malignancy & $3(11.5 \%)$ & $4(6.3 \%)$ \\
Musculoskeletal & $7(25.9 \%)$ & $9(14.1 \%)$ \\
Renal & $2(7.7 \%)$ & $2(3.1 \%)$ \\
\hline Other respiratory & $1(3.8 \%)$ & $3(4.7 \%)$ \\
None & $3(11.5 \%)$ & $4(6.3 \%)$ \\
\hline
\end{tabular}

at least five times per week, on different days. The PP analysis population was defined as participants who, on average, took up the offer of at least one face-to-face session per week or accessed the online programme at least once per week.

Baseline characteristics were summarised by randomisation group as means and SDs (continuous normally distributed variables), medians and IQRs (non-normally distributed variables) and frequencies and percentages (categorical variables). The mean differences in the outcomes between the intervention and control arms and 95\% and 90\% CIs were estimated using linear regression adjusted for disease severity measured by $\mathrm{FEV}_{1} \%$ predicted and baseline functional capacity (6MWT) as both factors are measurable and may impact on the response to exercise training. Residual analysis was performed to examine model assumptions.

\section{RESULTS}

\section{Recruitment and baseline characteristics}

Overall, 143 subjects were screened for eligibility. The trial ran from September 2015 to March 2016. Figure 1 shows the subject flow for screening, randomisation and follow-up in the study. Table 1 illustrates the personal characteristics and baseline measures for the randomised 90 patients. No important imbalances were identified for these variables between the two intervention groups. The 90 participants with COPD had a mean age of 70 years (8.2) and moderate airflow obstruction with a mean $\mathrm{FEV}_{1} \%$ predicted of $59 \%$ (22). Patients in intervention arms were well matched prior to rehabilitation. Comorbidities for each intervention arm are illustrated in table 2.

\section{Primary outcomes}

The baseline 6MWT distance was 416.5 (118.3) $\mathrm{m}$ in the face-to-face group and 388.7 (104.4) $\mathrm{m}$ in the online intervention group and rose to 445.1 (124.9) and 433.6 (102.9) $\mathrm{m}$, respectively after the intervention.

The adjusted mean difference for the 6MWT between groups for the ITT population was $23.8 \mathrm{~m}$ with the lower $95 \% \mathrm{CI}$ well above the non-inferiority threshold of -40.5 $\mathrm{m}$ at $-4.5 \mathrm{~m}$ with an upper $95 \% \mathrm{CI}$ of $+52.2 \mathrm{~m}$. This result was consistent in the PP population with a mean adjusted difference of $15 \mathrm{~m}$ (-13.7 to 43.8). Non-inferiority of intervention was seen whether the minimally clinically important difference (MCID) of 54 or $30 \mathrm{~m}$ was used (see figure 2A).

The CAT score difference in the ITT was -1.0 in favour of the online intervention with the upper $95 \%$ CI well below the non-inferiority threshold of 1.8 at 0.86 and a lower $95 \%$ CI of -0.2 .9 . The PP analysis was consistent with the ITT with a mean CAT score difference of -0.64 (95\% CI -2.5 to 1.2) (figure 2B).

\section{Secondary outcomes}

HADS recorded at baseline demonstrated a reduction indicative of improvement in both intervention arms. The 


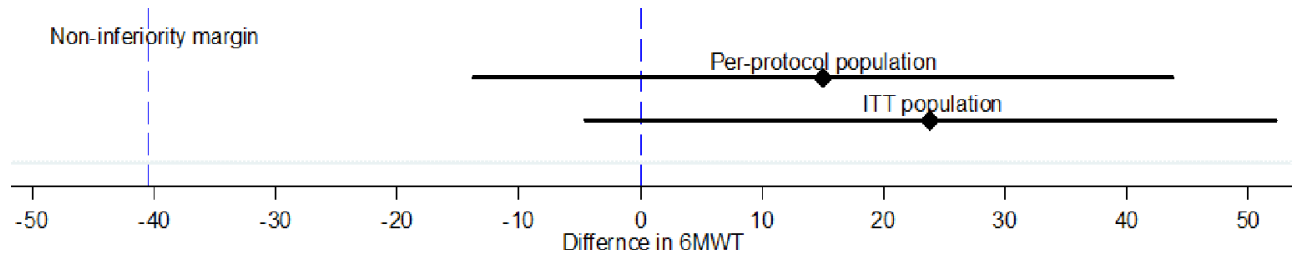

Figure 2A Adjusted mean difference and $95 \% \mathrm{Cl}$ for 6 min walk test (6MWT) in the intention-to-treat (ITT) and per-protocol (PP) population.

adjusted mean difference for HADS for the ITT population was -0.74 (95\% CI -3.5 to 0.9$)$ in favour of online PR. Similarly, COPD health-related quality of life (SGRQ) scores and mMRC dyspnoea scores suggested non-inferiority for the online intervention group (see table 3 ).

\section{PP analysis}

Fifteen (23\%) participants withdrew or were lost to follow-up from the online group and $5(19 \%)$ in the face-to-face treatment groups. A breakdown of the non-completer participants is summarised in the online supplementary Table S1. A further three participants had an exacerbation in the online group and were unable to complete the final study assessments. A PP analysis of all outcome measures recorded demonstrated differences well outside the clinically important difference for inferiority for all stipulated MCID values. All intervention effect estimates were in the direction of favour for the online intervention (see table 4).

\section{Safety}

Adverse events are summarised in table 5. Overall, both interventions were well tolerated with no safety issues identified.

\section{Adherence to rehabilitation training and education}

Adherence in both study arms was incomplete. Table 6 summarises the exercise sessions completed: a) attendance at the face-to-face group and b) participation with the online sessions. Overall, $72 \%$ of the two faceto-face sessions were attended, compared with $62 \%$ of the suggested five sessions recorded as accessed online over the 6-week intervention period. The attendance at the face-to-face sessions was relatively stable with a mean 1.6 sessions per participant in week 1 and 1.4 in week 6 , while there was a decline in participation in the online arm from a mean of 3.9 sessions per participant in week 1 to 2.5 in week 6 .

\section{DISCUSSION}

We report a single-blinded, randomised clinical trial of a novel and newly designed online pulmonary rehabilitation programme compared with the usual standard of care PR, delivered by face-to-face supervised patient sessions. The trial was designed to provide preliminary evidence for the use of online PR by examining the performance with respect to non-inferiority on validated clinical measures namely the 6MWT and the CAT score. The results are supportive of the hypothesis that there is no difference in either coprimary outcomes between these two approaches to delivering PR. In addition, non-inferiority was demonstrated between the impacts of online and conventional PR on validated clinical scores for breathlessness or health-related quality of life between the groups after the 6-week intervention period.

A predetermined PP analysis confirmed that for compliers the online PR was non-inferior with a direction of estimate in favour of online PR for all measures.

\section{Clinical improvements with pulmonary rehabilitation and comparison with other studies}

Pulmonary rehabilitation is part of standard care for patients with COPD who are functionally limited. ${ }^{12}$ It has been demonstrated to improve exercise tolerance and functional independence. ${ }^{3}$ The majority of studies of PR in COPD have demonstrated benefits through delivery of the model of a complex intervention of graded exercise and education over a 6-week course, which has now formed part of guideline-based treatment. ${ }^{2}$ The impact of conventional PR on key outcomes such as 6MWD test and CAT score has been assessed by a number of groups. In a meta-analysis of 14 studies which measured changes in the 6MWD test, the beneficial impact of PR was $55.7 \mathrm{~m}$ (27.8-92.8), ${ }^{20}$ with an MCID modelled at an improvement of $54 \mathrm{~m}$ for a patient to detect a benefit. ${ }^{14}$ Subsequent studies have identified $30 \mathrm{~m}$ as an appropriate value. ${ }^{16}{ }^{17}$

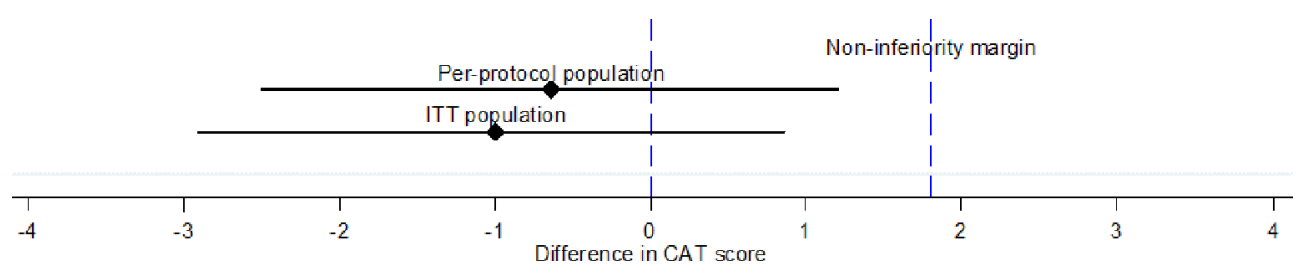

Figure 2B Adjusted mean difference and 95\% $\mathrm{Cl}$ for COPD assessment test (CAT) score in the intention-to-treat (ITT) and perprotocol (PP) population. 
Table 3 Between-group differences in primary and secondary outcomes

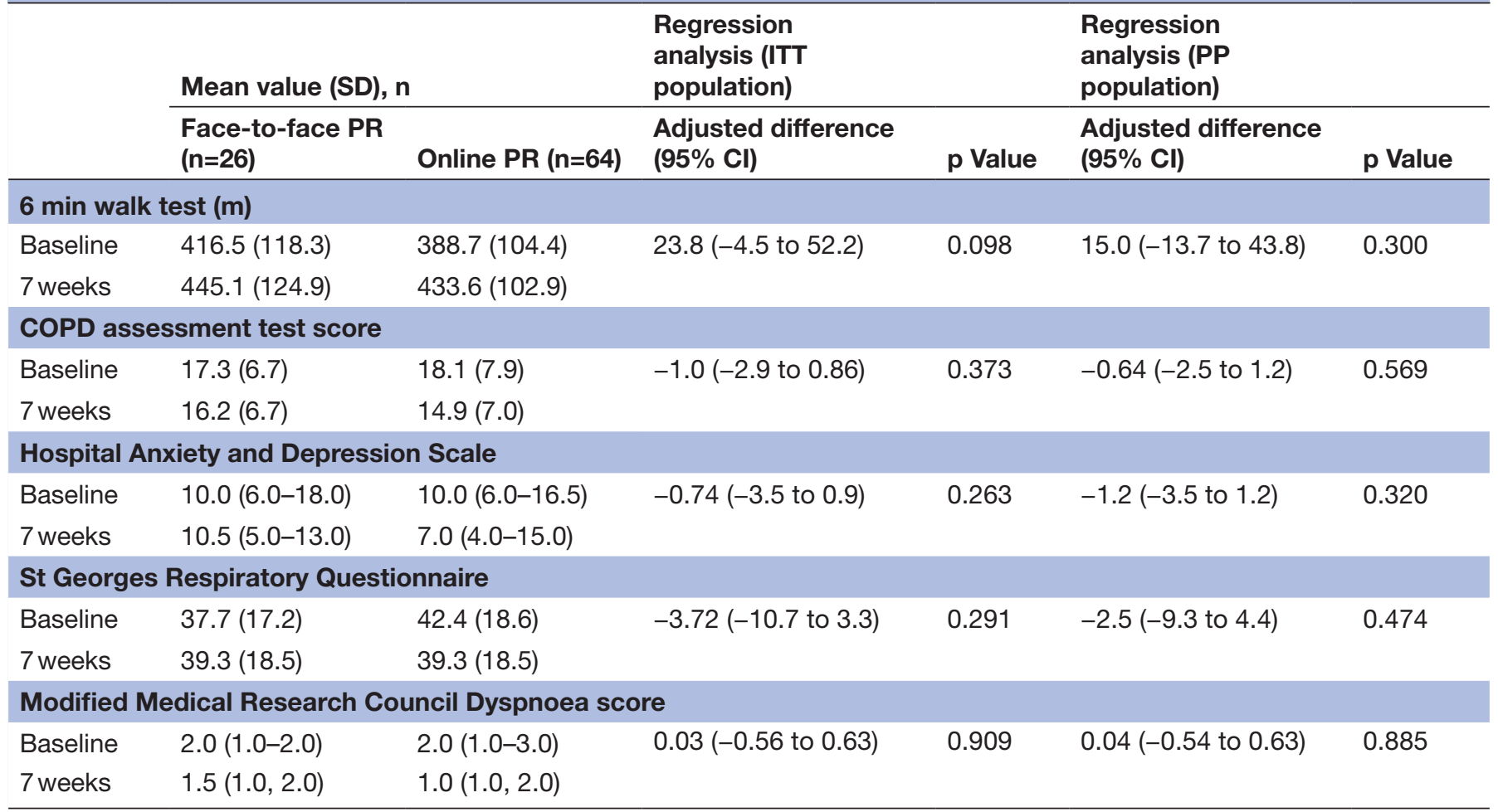

ITT, intention to treat; PP, per -protocol; PR, pulmonary rehabilitation.

The improvements in exercise capacity seen in this study in both treatment arms were within range of those in published analysis, demonstrated non-inferiority with both MCID values and were similar to small studies in comparable clinical groups. $^{21}$
While the evidence for safety and benefit of PR for patients with stable COPD is well established, the evidence behind the best methods to deliver this intervention is much less strong. The optimal duration of intervention has been established by clinical studies comparing length

Table 4 Number of participants with missing outcome data and summary by intervention arm for completers only

\begin{tabular}{|c|c|c|c|c|c|}
\hline & \multicolumn{5}{|l|}{ Mean value (SD), $n$} \\
\hline & Face-to-face PR & Online PR & Regression & p Value & Direction of estimate \\
\hline \multicolumn{6}{|c|}{6 min walk test } \\
\hline 7 weeks & 457.3 (122.1), 21 & 449.4 (99.0), 46 & & & \\
\hline \multicolumn{6}{|c|}{ COPD assessment test score } \\
\hline 7 weeks & $15.2(6.9), 21$ & $15.2(7.6), 44$ & & & \\
\hline \multicolumn{6}{|c|}{ Hospital Anxiety and Depression Scale } \\
\hline Baseline" & 10.0 (6.0-18.0), 26 & 10.0 (6.0-16.5), 64 & $-1.2(-3.3$ to 1.0$)$ & 0.267 & In favour of online PR \\
\hline 7 weeks & $10.0(4.5-12.5), 20$ & $6.5(4.0-14.5), 44$ & & & \\
\hline \multicolumn{6}{|c|}{ St Georges Respiratory Questionnaire } \\
\hline \multicolumn{6}{|c|}{ Modified Medical Research Council Dyspnoea score } \\
\hline Baseline & 2.0 (1.0-2.0), 26 & 2.0 (1.0-3.0), 64 & $-0.03(-0.55$ to 0.49$)$ & 0.912 & In favour of online PR \\
\hline 7 weeks & $1.0(1.0,2.0), 21$ & $1.0(1.0-2.0), 44$ & & & \\
\hline
\end{tabular}

PR, pulmonary rehabilitation 
Table 5 Intervention emergent adverse events by treatment groups

\begin{tabular}{lll}
\hline Adverse event & $\begin{array}{l}\text { Face-to-face } \\
\text { PR, } \mathbf{n}\end{array}$ & $\begin{array}{l}\text { Online } \\
\text { PR, } \mathbf{n}\end{array}$ \\
\hline Back pain & 1 & 1 \\
Muscular skeletal chest pain & 0 & 1 \\
Inguinal pain & 1 & 0 \\
Common cold & 1 & 0 \\
\hline
\end{tabular}

$\mathrm{PR}$, pulmonary rehabilitation

of PR exposure, ${ }^{22}{ }^{23}$ but the current design and delivery of education and exercise interventions is based largely on best practice and expert opinion. ${ }^{2}$ Indeed, the recent BTS guidelines on P R highlight the need for fundamental research in this area: 'The optimal structure of PR remains unknown. More robust studies are required to determine quality, cost-effectiveness and greater choice of delivery. To improve accessibility to PR, such research may include technologies'.

This study has established the potential for delivery of PR via an online platform in demonstrating non-inferiority of all measured outcomes compared with conventional PR.

Despite the widespread use of online technologies to manage almost every aspect of daily life, there are surprisingly few well-conducted clinical trials in this field. A small pilot study explored the use of online PR in COPD and found the intervention to hold possible merit with improvements in quality of life and a favourable cost-benefit model. ${ }^{24}$ Our approach supports this preliminary finding and offers new evidence that online-supported PR may benefit a range of patients with COPD who may be able to access this important intervention through the use of this technology for the first time.

Within the limitations of the sample size, our study demonstrated that online PR demonstrated no significant safety concerns and similarly to conventional PR appears to be an appropriate intervention if careful clinical measures are taken to mitigate risk. ${ }^{12}$ Significant numbers of patients were excluded due to exercise-induced oxygen desaturation. In face-to-face PR, supplemental oxygen can be administered and saturations monitored so for this subset of patients further work is required to ascertain the suitability of online-supported models and best practice therein.

\section{Access and adherence-key issues for delivery of PR}

Access to high-quality $\mathrm{PR}$ for patients with COPD is variable in the UK. ${ }^{25}$ Resource limitations, geographical distance from treatment centres and availability of classes which suit time commitments for participants have all been cited as key reasons why PR is currently ineffectively delivered to a large proportion of patients who may benefit. ${ }^{25}$ This national audit of PR services identified that over $37 \%$ of patients wait over 3 months for access to classes. Working patients are particularly disadvantaged as classes are often only provided during office hours. Consequently, attendance at PR is uniformly low and completion of courses similarly suboptimal with only $69 \%$ of patients referred attending for assessment. Capacity for delivery is currently limited - the UK National Audit estimates that 81000 referrals are made for PR each year-the great majority for COPD. This is in comparison to the estimated 900000 patients with an established diagnosis in the UK with a significant number of patients having no access to local services within a reasonable travelling time. ${ }^{225}$

Table 6 Exercise sessions completed by face-to-face $(n=26)$ and online groups $(n=64)$

\begin{tabular}{|c|c|c|c|c|c|c|}
\hline & Week 1 & Week 2 & Week 3 & Week 4 & Week 5 & Week 6 \\
\hline 0 sessions & $3(11)$ & $8(31)$ & 5 (19) & $6(23)$ & $6(23)$ & $7(27)$ \\
\hline Two sessions & $20(77)$ & $15(58)$ & $17(65)$ & $19(73)$ & $15(58)$ & $18(69)$ \\
\hline Mean & 1.6 & 1.3 & 1.5 & 1.5 & 1.3 & 1.4 \\
\hline 0 sessions & $9(14)$ & 12 (19) & $13(20)$ & $14(22)$ & $18(28)$ & $18(28)$ \\
\hline One session & $2(3)$ & $2(3)$ & $4(6)$ & $6(9)$ & $2(3)$ & $4(6)$ \\
\hline Two sessions & $6(9)$ & $5(8)$ & $7(11)$ & $8(13)$ & $6(9)$ & $11(17)$ \\
\hline Three sessions & $4(6)$ & $7(11)$ & $5(8)$ & $8(13)$ & $11(17)$ & $8(13)$ \\
\hline Seven sessions & $4(6)$ & $1(2)$ & $0(0)$ & $2(3)$ & $1(2)$ & $0(0)$ \\
\hline Mean & 3.9 & 3.5 & 3.2 & 3.0 & 2.8 & 2.5 \\
\hline
\end{tabular}

All numbers are $\mathrm{n}(\%)$ 
The majority of patients in high-income countries have access to the internet-a recent study established that over $86 \%$ of patients with cardiopulmonary disease have direct access. ${ }^{11}$ Indeed, the majority of subjects (161 of 163) assessed for eligibility for the study had access to the internet. Furthermore, study of regular internet use in patients with long-term conditions has identified that the majority with access (68\%), use the internet regularly to understand more about their disease. ${ }^{26}$ Currently, disease relevant information is often available from charities and patient group sites and users seldom have access to prescribed interventions to date. This situation will undoubtedly change rapidly in the UK with the announcement that the National Health Service (NHS) will support prescription of digital health technologies from April 2017. However, even with improving patterns of internet access it is important that most if not all patients can use digital platforms effectively and with minimum requirements for training and support The 'myCOPD' web app was designed with patients and extensive usability assessments were carried out in the development process. Inevitably, even with a user-friendly system implementation to all patients with a chronic condition will be challenging, further studies to define optimal models to ensure equity of access are required and resources to ensure support considered in health economic assessments.

Adherence to PR is another barrier to its overall impact in this patient group. In 1998, Singh et al found less than half of patients referred to PR completed the course. ${ }^{27}$ Adherence rates reported in clinical trials such as this tend to be higher and our completion rates of over $70 \%$ for this trial are in line with these. There is minimal published evidence regarding the factors that lead to non-adherence to PR in patients with COPD. Young et al identified a number of patient factors including social isolation and active smoking which predicted poor adherence to PR, suggesting that the necessity for patients to participate in group sessions may be a barrier for certain patients. ${ }^{28}$ In our study, adherence to the intervention in both arms was as expected, incomplete. Although there was an attrition over the 6 -week intervention period to the use of online PR, in terms of supported sessions accessed each week, the mean was still greater than the face-toface arm. Very little additional support from the trial or technical team was required in this study by patients in the online arm, with most issues resolved remotely. It is possible a more intensive online or telephone mentoring approach may improve adherence yet further. We suggest further studies are required to determine the patient's preference for the model of access to PR and the impacts of this 'patient-centred' approach on access and adherence.

\section{Comparison with digital health interventions in other disease areas}

In other disease areas or aspects of COPD care, there is a richer evidence base to support the role of digitally supported interventions. In the management of dyspnoea, a comparison of internet-based versus face-toface supported self- management in COPD was assessed in a small randomised controlled trial. ${ }^{29}$ This study was published over 8 years ago and was troubled by technical challenges; however, its findings demonstrated that both online and face-to-face programmes were useful in improving dyspnoea. Cardiac rehabilitation is another evidence-based facet of the management of a long-term condition. Patients with cardiac conditions demonstrate a high level of interest in the concept of technology-enabled home rehabilitation. ${ }^{30}$ Clinical studies of internet-based interventions suggest clinical benefit for patients with ischaemic heart disease, although overall conclusions are limited by poor trial quality and the data to support improvements in activity was stronger. ${ }^{31}$

The data from our study is aligned with the available data from existing trials across a range of diseases suggesting that online supported management and rehabilitation may offer clinical benefits. Considering the range of comorbidities that a large proportion of patients with long-term conditions suffer from it is vital that a coordinated approach to enable an overall improvement in health rather than just single disease relevant outcomes is the goal for the emerging use of mobile health technologies. This will require close working between clinicians, technologists and commissioners to ensure that a coordinated and patient friendly approach is developed along with rigorous testing to establish clinical benefit and cost-effectiveness.

\section{Limitations of this study}

We acknowledge a number of limitations to the interpretation of this trial. It is a relatively small study, which was designed to explore the non-inferiority of online PR intervention. While we report that all clinical outcomes were non-inferior, we accept that a larger randomised controlled trial fully powered to demonstrate health economic benefits is required to explore the potential to change the model of PR delivery and hence clinical practice. The study was relatively short-in line with the current clinical model of 6-week to 12-week clinical PR courses. As extending the online intervention is not limited by resource implications, it will be possible to explore the role of long-term programmes including maintenance classes and the duration of impacts using this model. Our study was also delivered at a single centre; we recognise practice may differ across providers and regions and hence a multicentred pragmatic study is indicated to understand the place for online PR in complimenting current practice in a range of clinical settings.

As with all studies of exercise-related interventions, double blinding was not possible; however, this will have impacted on both groups. Every effort was made to ensure assessments were made in a blinded fashion in both arms. As many of the barriers to delivery of face-to-face PRaccess, geography and capacity-are overcome within the conduct of a randomised controlled trial, it is likely that the real test of online technologies such as this will be against 
the usual standard of care and so 'real-world' data will be key to explore the potential uptake and impacts in this situation. Therefore, further work is needed-not to establish comparative efficacy of models but to establish a real-world evidence base and to understand the long-term utility of scalable digital platforms across healthcare settings.

\section{Comparison with current guidelines}

National and international guidelines recommend PR for patients with COPD with functional limitation. ${ }^{12}$ Current models for delivering PR are based on best practice advice and rely on a model of face-to-face delivery, which has been established over a number of years. Our study was designed and delivered in this context and provides important evidence that a new model of internet-enabled delivery for this vital intervention may be considered by clinicians.

\section{Conclusions and policy implementations}

COPD is a disease of global health importance with a limited array of clinically proven interventions available to clinicians or patients to improve outcomes, PR is one of these interventions and has become part of the standard of care for this disease. Recent national audits have identified significant inadequacies in accessing PR and recent UK guidelines identify the need for novel studies to explore new models of delivering PR to patients with COPD to overcome this unmet need.

We have conducted a significant study to explore non-inferiority of the role of internet-enabled PR to improve clinical outcomes compared with the standard model of clinical delivery. We have demonstrated for the first time that in all clinical measures studied, online PR using the myMHealth platform is non-inferior to usual care and suggest that this modality of delivery be explored widely in the delivery of this important intervention in this common disease. There is now a potential opportunity for the online provision of PR to compliment currently available face-to-face services in order to increase capacity, reduce costs and broaden availability to socially or geographically isolated groups, which requires exploration in future studies across wide populations to establish optimal implementation of strategies and to assess health economic benefits.

Acknowledgements We thank the clinical trials team at Queen Alexandra Hospitals NHS trust and the staff of myMHealth. We are grateful to the patients who contributed to the design and conduct of the study.

Contributors RDV, MN, SCB, VC and TW contributed to the study design. BG was principal investigator. RDV, MN, BG, TB and AJC contributed to the study delivery. VC analysed the data. All authors contributed to data interpretation and manuscript preparation and reviewed the final draft. TW is guarantor for the data.

Funding The study was funded by a Small Business Research Initiative (SBRI) grant from NHS England.

Competing interests Dr Bourne reports grants and personal fees from myMHealth (a medical software company) during the conduct of the study; other from myMHealth, outside the submitted work. He is CEO, co-founder and part owner of this company. Mrs De Vos reports personal fees from myMHealth, during the conduct of the study; and is a partner in the rehabilitation facility that hosted some of the clinical trial activity. Dr Green reports grants to Portsmouth Hospitals NHS
Trust from myMHealth, during the conduct of the study. Mr North has nothing to disclose. Dr Cornelius reports personal fees from myMHealth, during the conduct of the study. Professor Chauhan has nothing to disclose. Dr Brown reports grants from myMHealth, during the conduct of the study. Professor Wilkinson reports grants and personal fees from myMHealth during the conduct of the study. He is co-founder and part owner of this company.

Patient consent Detail has been removed from this case description/these case descriptions to ensure anonymity. The editors and reviewers have seen the detailed information available and are satisfied that the information backs up the case the authors are making.

Ethics approval This study was approved by the research ethics committee for Berkshire B of the UK Health Research Authority (15/SC/0345).

Provenance and peer review Not commissioned; externally peer reviewed.

Data sharing statement All anonymised trial data are available on application to the senior author.

Open Access This is an Open Access article distributed in accordance with the Creative Commons Attribution Non Commercial (CC BY-NC 4.0) license, which permits others to distribute, remix, adapt, build upon this work non-commercially, and license their derivative works on different terms, provided the original work is properly cited and the use is non-commercial. See: http://creativecommons.org/ licenses/by-nc/4.0/

(c) Article author(s) (or their employer(s) unless otherwise stated in the text of the article) 2017. All rights reserved. No commercial use is permitted unless otherwise expressly granted.

\section{REFERENCES}

1. National Institute for Health and Care Excellence. Chronic obstructive pulmonary disease;. 2013 https://www.nice.org.uk/guidance/ conditions-and-diseases/respiratory-conditions/chronic-obstructivepulmonary-disease.

2. Society BT. British thoracic society quality standards for pulmonary rehabilitation in adults, 20146https://www.brit-thoracic.org.uk/ document-library/clinical-information/pulmonary-rehabilitation/btsquality-standards-for-pulmonary-rehabilitation-in-adults/.

3. McCarthy B, Casey D, Devane D, et al. Pulmonary rehabilitation for chronic obstructive pulmonary disease. Cochrane Database Syst Rev 2015:CD003793.

4. Garcia-Aymerich J, Lange P, Benet M, et al. Regular physical activity reduces hospital admission and mortality in chronic obstructive pulmonary disease: a population based cohort study. Thorax 2006;61:772-8.

5. Fischer MJ, Scharloo M, Abbink JJ, et al. Drop-out and attendance in pulmonary rehabilitation: the role of clinical and psychosocial variables. Respir Med 2009;103:1564-71.

6. Arnold E, Bruton A, Ellis-Hill C. Adherence to pulmonary rehabilitation: a qualitative study. Respir Med 2006;100:1716-23.

7. Vogiatzis I, Rochester CL, Spruit MA, et al. Increasing implementation and delivery of pulmonary rehabilitation: key messages from the new ATS/ERS policy statement. Eur Respir J 2016;47:1336-41 http:// www.ncbi.nlm.nih.gov/pubmed/27132269.

8. Mendes de Oliveira JC. Studart Leitao Filho FS, Malosa Sampaio LM, Negrinho de Oliveira AC, Hirata R, Costa D, Donner CF, de Oliveira LV. outpatient vs. Home-based pulmonary rehabilitation in COPD: a randomized controlled trial. Multidisciplinary Respiratory Medicine 2010;5:401.

9. Holland AE, Hill CJ, Rochford P, et al. Telerehabilitation for people with chronic obstructive pulmonary disease: feasibility of a simple, real time model of supervised exercise training. J Telemed Telecare 2013;19:222-6.

10. Cruz J, Brooks D, Marques A. Home telemonitoring effectiveness in COPD: a systematic review. Int J Clin Pract 2014;68:369-78.

11. Disler RT, Inglis SC, Newton PJ, et al. Patterns of technology use in patients attending a cardiopulmonary outpatient clinic: a self-report survey. Interact J Med Res 2015;4:e5.

12. Podsiadlo D, Richardson S. The timed "Up \& Go": a test of basic functional mobility for frail elderly persons. J Am Geriatr Soc 1991;39:142-8.

13. Mesquita R, Wilke S, Smid D, et al. Timed up \& go test in COPD: changes over time. validity and responsiveness to pulmonary rehabilitation. 1.2 Rehabilitation and Chronic Care 2014;44(Suppl 58):3037. Sep.

14. Redelmeier DA, Bayoumi AM, Goldstein RS, et al. Interpreting small differences in functional status: the six Minute walk test in chronic lung disease patients. Am J Respir Crit Care Med 1997;155:1278-82. 
15. Troosters $T$, Gosselink R, Decramer M, et al. Six minute walking distance in healthy elderly subjects. Eur Respir J 1999;14:270-4.

16. Puhan MA, Chandra D, Mosenifar Z, et al. The minimal important difference of exercise tests in severe COPD. Eur Respir $J$ 2011;37:784-90.

17. Holland AE, Hill CJ, Rasekaba T, et al. Updating the minimal important difference for six-minute walk distance in patients with chronic obstructive pulmonary disease. Arch Phys Med Rehabil 2010;91:221-5.

18. GOLD- Global Initiative for Chronic Obstructive lung disease. https:// www.guidelines.co.uk/gold/copd.

19. Royston P. Multiple imputation of missing values: update. The Stata Journal 2005;5:1-14

20. Lacasse $\mathrm{Y}$, Wong $\mathrm{E}$, Guyatt GH, et al. Meta-analysis of respiratory rehabilitation in chronic obstructive pulmonary disease. Lancet 1996;348:1115-9.

21. Laviolette L, Bourbeau J, Bernard S, et al. Assessing the impact of pulmonary rehabilitation on functional status in COPD. Thorax 2008;63:115-21.

22. Beauchamp MK, Janaudis-Ferreira T, Goldstein RS, et al. Optimal duration of pulmonary rehabilitation for individuals with chronic obstructive pulmonary disease - a systematic review. Chron Respir Dis 2011;8:129-40.

23. Green $\mathrm{RH}$, Singh SJ, Williams J, et al. A randomised controlled trial of four weeks versus seven weeks of pulmonary rehabilitation in chronic obstructive pulmonary disease. Thorax 2001;56:143-5.

24. Burkow TM, Vognild LK, Johnsen E, et al. Comprehensive pulmonary rehabilitation in home-based online groups: a mixed method pilot study in COPD. BMC Res Notes 2015;8:766.

25. Steiner M, Holzhauer-Barrie J, Lowe D, et al; Pulmonary Rehabilitation: time to breath better. Resources and organisation of
Pulmonary Rehabilitation services in England and Wales: National Chronic Obstructive Pulmonary Disease (COPD) Audit Programme, 2015. www.rcplondon.ac.uk/COPD.

26. Martinez $\mathrm{CH}$, St Jean $\mathrm{BL}$, Plauschinat $\mathrm{CA}$, et al. Internet access and use by COPD patients in the National Emphysema/COPD Association survey. BMC Pulm Med 2014;14:66.

27. Singh SJ, Smith DL, Hyland ME, et al. A short outpatient pulmonary rehabilitation programme: immediate and longer-term effects on exercise performance and quality of life. Respir Med 1998;92:1146-54.

28. Young P, Dewse M, Fergusson W, et al. Respiratory rehabilitation in chronic obstructive pulmonary disease: predictors of nonadherence. Eur Respir J 1999;13:855-9.

29. Nguyen HQ, Donesky-Cuenco D, Wolpin S, et al. Randomized controlled trial of an internet-based versus face-to-face dyspnea self-management program for patients with chronic obstructive pulmonary disease: pilot study. J Med Internet Res 2008;10:e9.

30. Buys R, Claes J, Walsh D, et al. Cardiac patients show high interest in technology enabled cardiovascular rehabilitation. BMC Med Inform Decis Mak 2016;16:95.

31. Devi R, Singh SJ, Powell J, et al. Internet-based interventions for the secondary prevention of coronary heart disease. Cochrane Database Syst Rev 2015;22:CD009386.

32. Cote CG, Casanova C, Marín JM, et al. Validation and comparison of reference equations for the 6-min walk distance test. Eur Respir $J$ 2008;31:571-8

33. Kon SS, Canavan JL, Jones SE, et al. Minimum clinically important difference for the COPD Assessment Test: a prospective analysis. Lancet Respir Med 2014;2:195-203. 